\title{
ATRIBUCIONES CAUSALES EXPLÍCITAS E IMPLÍCITAS EN EL DELIRIO: ESTUDIO DE SU ESPECIFICIDAD EN DELIRIOS PARANOIDES Y NO PARANOIDES
}

\author{
Carmelo Vazquez ${ }^{1}$, Cristina díez-Alegria ${ }^{2}$, Marta Nieto-Moreno ${ }^{3}$, \\ CARMEN VALIENTE ${ }^{2}$ Y FILIBERTO FUENTENEBRO ${ }^{2}$ \\ 'Facultad de Psicología, Universidad Complutense de Madrid \\ ${ }^{2}$ Servicio de Psiquiatría, Hospital Clínico Universitario San Carlos, Madrid \\ ${ }^{3}$ Departamento de Psiquiatría. Universidad Autónoma de Madrid
}

\begin{abstract}
Resumen: En este trabajo se estudian sesgos cognitivos atribucionales en pacientes delirantes. Para analizar el estilo atribucional, se emplean tareas de evaluación de razonamiento causal explícitas (IPSAQ) e implícitas (PIT) en una muestra de 40 pacientes delirantes (19 paranoides y 21 no paranoides). Los resultados indican la presencia de sesgos atribucionales (i.e. externalizante y personalizante) en pacientes delirantes con independencia del tipo de delirio cuando se emplea una medida de evaluación explícita. Sin embargo, el patrón atribucional implícito de los pacientes con delirios paranoides es diferente al mostrado por los pacientes no paranoides. Se discuten las implicaciones teóricas y clínicas de estos hallazgos.
\end{abstract}

Palabras clave: Delirios, paranoide, sesgos cognitivos, atribuciones, razonamiento causal, tareas explícitas, tareas implicitas.

\section{Explicit and implicit causal attributions in delusions: A study of its specificity in paranoid delusions vs non paranoid delusions}

\begin{abstract}
In this paper the presence of atributional cognitive biases in delusional patients is studied. Explicit (IPSAQ) and implicit (PIT) attributional measures have been used to analyze the attributional style in a sample of 40 delusional patients ( 19 paranoid patients and 21 non paranoid patients). The results showed the presence of attributional biases (i.e. externalizing and personalizing) independently of the delusional content when an explicit task was used. Compared to non paranoid patients, the paranoid group showed a different attributional style when it was assessed through an implicit task. Theoretical and clinical implications of the results are discussed.
\end{abstract}

Key words: Delusions, paranoid, cognitive biases, attributions, causal reasoning, explicit tasks, implicit tasks.

\section{INTRODUCCIÓN}

La presencia de delirios ha sido un típico criterio indicativo de un trastorno mental grave. A pesar de ser uno de los síntomas más comunes y un «tema primordial» de la psicopatología y clí-

Recibido: 23 de junio de 2005. Aceptado: 12 de diciembre de 2005.

Correspondencia: Carmelo Vázquez, Facultad de Psicología, Universidad Complutense de Madrid, Campus de Somosaguas, 28223-Madrid (España). Tfno.:(34) 91-3943131. Fax: (34) 913943189. Correo-e: cvazquez@psi.ucm.es.

Esta investigación fue, en parte, subvencionada por el Ministerio de Ciencia y Tecnología (BSO2003-07516), la Fundación Lafourcade-Colegio Oficial de Psicólogos, y apoyada por una beca FPU-MEC (Ref 2001-0786) concedida a Marta NietoMoreno. nica psiquiátrica (Ey, 1950), su investigación como sintoma aislado ha sido asombrosamente escasa durante décadas, contrastando con la proliferación de investigaciones sobre cuadros clinicos que, paradójicamente, son en parte definidos por la presencia de aquél (p.ej. esquizofrenia, trastornos afectivos con sintomatología psicótica, psicosis tóxicas, trastornos delirantes, etc.) —Vázquez, Valiente y Díez-Alegría, 1999.

\section{Estudio de sintomas en psicopatologia experimental}

Tradicionalmente, tanto el trabajo de los clínicos como el de aquellos dedicados a la inves- 
tigación en el ámbito de la Psicopatología se ha guiado por los conocidos sistemas clasificatorios como el DSM (APA) y la CIE (OMS). Durante las dos últimas décadas estos procedimientos clasificatorios de la conducta «patológica» se han puesto en entredicho debido a las limitaciones conceptuales y metodológicas que presentan. En esta línea, son muchos los investigadores que han señalado las dificultades que conlleva el estudio de un grupo de sujetos «etiquetados» bajo la misma categoría diagnóstica que, pese a su aceptable fiabilidad, presenta importantes problemas de validez (concurrente, de constructo y predictiva) que, además de sesgar los hallazgos de los estudios, pueden repercutir directamente en la práctica clínica (Costello, 1992).

Ante la necesidad de adoptar nuevas estrategias de estudio, en las últimas décadas ha surgido un fuerte interés por la investigación centrada en síntomas específicos, paradigma de estudio que cuenta con diversas ventajas como evitar problemas de las categorías diagnósticas y de clasificación, permitir centrarse en fenómenos habitualmente ignorados (como ha sido el caso de los delirios), mejorar la comprensión de la fenomenología de síntomas particulares, facilitar desarrollos teóricos, permitir la identificación de fenómenos clínicos relacionados con el comportamiento normal, mejorar la capacidad discriminativa de los sistemas de clasificación derivada de una mejor comprensión de sintomas individuales y facilitar la identificación de síntomas específicos como objetivos de las intervenciones terapéuticas (Persons, 1986). Así, la «vuelta al síntoma» como objeto de estudio en la psicopatología experimental solventa problemas de fiabilidad y sobre todo los problemas de validez de las clasificaciones diagnósticas, permitiendo centrar nuestra atención en fenómenos de enorme interés. De este modo, se potencia el estudio multifactorial y multidimensional del síntoma, así como la perspectiva de continuidad patología — normalidad más adecuada que las aproximaciones psiquiátricas de tipo categorial (ausencia / presencia)Vázquez et al., 1999.

\section{Razonamiento causal y delirios}

La mayor parte de los delirios (paranoides, de control, de grandiosidad, de referencia, etc.) hacen referencia a la posición que el paciente adopta con respecto a los demás, así como a las intenciones que los otros tienen hacia él. Debido al «carácter social» de algunos síntomas, la Teoría de la Atribución Social de Kelly (1967) ha propiciado un fructífero marco de trabajo no sólo para el estudio de cuadros clínicos como la depresión, sino también para el estudio de los delirios, ya que da cuenta de cómo tratamos de explicar cuanto nos acontece, qué tipo de explicaciones causales damos a nuestra propia conducta y a la conducta de los demás (ver una revisión en Díaz-Alegría y Vázquez, en prensa).

Para el estudio de patrones de razonamiento causal tradicionalmente se han empleado tareas en las que se les pide al sujeto que infiera las causas de sucesos hipotéticos de distinta naturaleza, como el Attributional Style Questionnaire (ASQ; Peterson et al., 1982) o el ASQpf (Lyon, Kaney y Bentall, 1994). Los estudios que han empleado estas tareas han mostrado que los sujetos con delirios paranoides presentan un sesgo atribucional externalizante similar o incluso mayor que el de sujetos normales: ante sucesos positivos su estilo atribucional es interno y ante sucesos negativos externo, global y estable (Candido y Romney, 1990; Fear, Sharp y Healy, 1996; Kaney y Bentall, 1989; Díez-Alegría, Vázquez, Nieto, Valiente y Fuentenebro, en prensa), siendo este sesgo diametralmente opuesto al de pacientes depresivos, quienes tienden a atribuir a causas internas, globales y estables los sucesos negativos (Brewin, 1985; Mezulis, Abramson, Hyde, y Hankin, 2004).

Autores como White (1991) o Levenson (1974) han distinguido tres tipos de locus atribucional en la dimensión de internalidad: locus interno, locus externo-personal (atribuir la causa de lo que sucede a la acción u omisión de otros) y locus externo-situacional (atribuir las causas de lo que sucede a las circunstancias o al azar). El hecho de que la subescala de inter- 
nalidad del ASQ suela presentar bajos índices de fiabilidad en la dimensión internalidad (Reivich, 1995) propició el desarrollo de una nueva estrategia de evaluación de patrones de razonamiento causal, el Internal, Personal and Situational Attributions Questionnaire (IPSAQ; Kinderman y Bentall, 1996a) que además de solventar el problema de la fiabilidad permite distinguir los tres tipos de locus propuestos. El empleo de esta escala en el estudio de sesgos atribucionales en pacientes delirantes no sólo ha confirmado los hallazgos previos sino que además ha mostrado que los pacientes con delirios paranoides tienden a culpar a otra (s) persona (s) de los sucesos negativos (i.e. atribución externa de tipo personalizante) en lugar de atribuir las causas al azar, la situación o las circunstancias (atribución externa situacional) -Kinderman y Bentall, 1997.

Basándose en los resultados de investigaciones sobre sesgos cognitivos y perceptivos, el grupo de investigación de Bentall (Bentall, Kinderman y Kaney, 1994; Kinderman y Bentall, 1996b; Kinderman y Bentall, 1996c) propuso un modelo de razonamiento paranoide según el cual, este sesgo atribucional «exagerado» (i.e. tendencia a culpar a los demás de los eventos negativos) presente en los pacientes delirantes tendría la función de preservar la autoestima en la medida en que evita el acceso a auto-esquemas negativos. Ante un suceso negativo que podría suponer una amenaza a la autoestima (por ejemplo, ser despedido del trabajo), el paciente delirante atribuiría sus causas a aspectos externos relacionados con otras personas (por ejemplo, culparía a su jefe porque «le tiene envidia o manía») reforzando así la visión negativa que tiene sobre las intenciones de los demás y evitando, además, la activación de discrepancias entre cómo se ve actualmente a sí mismo de manera realista (autoconcepto actual o «Yo actual») y cómo le gustaría ser (autoconcepto ideal o "Yo ideal»). De esta forma, evitaría acceder a auto-esquemas negativos, que podrían suponer una disminución de su autoestima (Díez-Alegría y Vázquez, en prensa; McKay, Langdon, y Colheart, 2005). Así, la creencia paranoide estaría ligada a un autoconcep- to positivo explícito y cumpliría una función defensiva (idea planteada ya por Zigler y Glick, 1988).

Un aspecto de especial interés es el hecho de que el sesgo externalizante exagerado, sólo se muestra en pacientes delirantes cuando se emplean tareas de evaluación de razonamiento causal explícitas o directas, es decir, cuando los cuestionarios valoran directamente las explicaciones causales para un suceso hipotético dado. Algunos estudios que han empleado tareas implícitas para su evaluación, por ejemplo el Pragmatic Inferencial Test (PIT) - p.ej. Lyon, Kaney y Bentall, 1994 - han encontrado que los mismos pacientes delirantes que mostraban un sesgo auto-sirviente en tareas explícitas como el ASQ o el IPSAQ, mostraban sin embargo un estilo atribucional de tipo depresógeno cuando se exploraba con tareas de razonamiento causal implícitas. Este tipo de hallazgos podría estar indicando la existencia de patrones de razonamiento causal diferentes en función del tipo de medida empleada (i.e. explícita vs. implícita), lo que reflejaría la existencia de un auto-esquema defensivo en los pacientes delirantes (McKay, Langdon, y Colheart, 2006; Smith, Freeman y Kuipers, 2005).

\section{Limitaciones de los estudios sobre razonamiento causal y delirios}

Aunque la investigación psicológica sobre delirios ha sido muy fructífera, no está exenta de limitaciones. Entre éstas, cabe señalar la ausencia de especificidad de los hallazgos; muchos de los estudios han estudiado categorías diagnósticas tan amplias y heterogéneas como la esquizofrenia y, además, la gran mayoría de los trabajos han centrado su atención casi exclusivamente en los delirios paranoides (tras los delirios de referencia, es el sintoma más común en la esquizofrenia y el tipo de delirio más frecuente en los trastornos delirantes) - Sartorius et al. 1986; APA, 1996. Por estas razones, la extensión de los hallazgos a otro tipo de delirios (e.g. delirios de control) no está garantizada. De hecho, un estudio reciente de Smith et al. (2005) ha demostrado que en los pacientes con 
delirios de grandeza no se observa ninguna diferencia entre medidas explícitas de autoestima (medida con un cuestionario autoaplicado) e implícitas (i.e., autoestima evaluada con tareas atencionales Stroop y tareas de recuerdo incidental).

La consistencia de los hallazgos previos así como limitaciones como la anteriormente señalada, dejan la puerta abierta a estudios como el aquí planteado, cuyo objetivo fundamental fue determinar si los sesgos cognitivos atribucionales (i.e. sesgo externalizante, sesgo personalizante y sesgo auto-sirviente) eran específicos de un determinado tipo de delirio (paranoide o de persecución) o si por el contrario, estaban presentes en la sintomatología delirante con independencia de su contenido o temática. Como segundo objetivo nos planteamos estudiar la existencia de un estilo atribucional depresógeno subyacente en los delirios de tipo persecutorio (esto es, tendencia a culparse de sucesos negativos) que sólo se mostraría cuando se emplean tareas implicitas para su evaluación. En función de la literatura revisada nuestras principales hipótesis fueron que en tareas de evaluación explícita, los sesgos externalizante y personalizante estarian presentes tanto en delirios paranoides como en delirios no paranoides, habiendo diferencias únicamente en el sesgo personalizante, que sería de mayor magnitud en los delirios paranoides (esto es, un estilo menos autoinculpador ante sucesos negativos). En tareas de evaluación implícita, nuestra hipótesis fue que sólo en el caso de los delirios paranoides aparecería un estilo atribucional depresógeno. En delirios no paranoides no esperamos encontrar diferencias en el patrón de razonamiento causal ligadas al tipo de tarea empleada.

\section{MÉTODO}

\section{Participantes}

Siguiendo los procedimientos de la investigación centrada en el estudio de síntomas, participaron en el estudio 40 pacientes delirantes ( 27 hombres y 13 mujeres) con una edad media de 33,32 años (DT $=8,41)$ ingresados en el Ser- vicio de Psiquiatría del Hospital Clínico San Carlos de Madrid y en la Unidad de Hospitalización Breve del Hospital Psiquiátrico de Madrid. Aunque esta investigación se centró en el síntoma delirante y no partió de etiquetas diagnósticas categoriales, en términos de criterios diagnósticos DSM-IV (APA, 1996) 25 participantes cumplían de esquizofrenia paranoide, 1 de esquizofrenia indiferenciada, 6 de trastorno esquizoafectivo, 6 de trastorno psicótico no especificado y 2 de trastorno psicótico breve. Los criterios de inclusión fueron tener una edad comprendida entre los 18 y 65 años y presentar sintomatología delirante activa, según la definición de idea delirante del DSM-IV, en el momento de realizar el estudio con independencia del diagnóstico. Se excluyeron aquellos pacientes cuyo historial clínico reflejara la existencia de anomalías orgánicas, historia de uso / abuso de alcohol y drogas, así como aquéllos que cursaban con psicosis tóxicas. Además, se excluyeron los pacientes cuya temática delirante estuviera relacionada con ideas de culpa debido a que el solapamiento de este tipo de delirios con características depresivas pudiera sesgar significativamente los resultados obtenidos en las tareas de evaluación de razonamiento causal. En el momento de participar en el estudio, todos los participantes recibían medicación antipsicótica.

\section{Instrumentos de evaluación clínica}

Brief Psychiatry Rating Scale (BPRS). Es una entrevista semi-estructurada que evalúa gravedad de la sintomatología, especialmente psicótica, durante las dos semanas previas a su aplicación. Se empleó la versión de 24 ítems (Lukoff, Nuechterlein y Ventura, 1986), evaluados según la escala de gravedad de 1 (ausente) a 7 (muy grave). Además de una puntuación total, la BPRS proporciona información de siete factores: Ansiedad-Depresión, Anergia, Trastornos del Pensamiento, Activación, Hostilidad/Suspicacia, Factor Sintomas Positivos y Factor Sintomas Negativos. El alpha de Cronbach para la puntuación total del BPRS fue de $\alpha=0,76$. 
Maudsley Assesment of Delusional Schedule (MADS; Buchanan et al., 1933; Taylor et al., 1994). La MADS es una entrevista semiestructurada que evalúa la ideación delirante desde un punto de vista dimensional. Permite evaluar 8 dimensiones de la misma (Grado de convicción, Mantenimiento de la creencia, Afecto en relación con la creencia, Acciones relacionadas con la creencia, Idiosincrasia de la creencia, Grado de preocupación, Sistematización de la creencia e Insight) y ha demostrado buenos índices psicométricos de validación (Buchanan et al., 1993) -cit. Vázquez et al., 1999. En este trabajo, el MADS se utilizó para identificar el tipo de contenido de la creencia principal delirante de los participantes. Para la consecución de los objetivos propuestos, la muestra fue dividida en dos grupos. La variable de agrupación fue el tipo de delirio predominante en el momento en que se realizó la evaluación (paranoide vs. no paranoide), evaluado mediante esta entrevista estructurada. El empleo de esta estrategia de formación de los grupos, es relativamente novedosa en la medida en que difiere de otros procedimientos habitualmente utilizados por otras entrevistas diagnósticas más generales (p.ej. Present State Examination o SCID) las cuales se centran casi exclusivamente en la identificación del tipo de delirio desde una asunción categorial (i.e. delirio paranoide presente vs ausente). La ventaja añadida del uso del MADS es que permite el análisis cualitativo y narrativo de los contenidos delirantes verbalizados por el paciente, además de su análisis cuantitativo multidimensional. Para hacer el procedimiento de subclasificación de los delirios aún más fiable, se utilizaron otros criterios, desde la propia MADS, para determinar cuál era la principal creencia delirante (i.e., aquélla que en el momento de la evaluación era la que causaba mayor malestar al paciente, era la más intrusiva, o a la que más atención prestaba). En todos los casos existió concordancia total entre los evaluadores y el profesional de la salud de referencia del paciente en el hospital, respecto a la identificación del tipo de delirio predominante.
Beck Depression Inventory (BDI; Beck, Rush, Shaw y Emery, 1979). El BDI es un cuestionario formado por 21 items que evalúa gravedad de sintomatología depresiva según una escala de 0 a 3 . Sus índices de fiabilidad y validez en poblaciones clínicas y subclínicas son buenos y su especificidad adecuada (Sanz y Vázquez, 1998; Vázquez y Sanz, 1999). En este estudio, el indice de consistencia fue elevado $(\alpha=0,93)$.

Escala de Autoestima de Rosenberg (RSEQ; Rosenberg, 1965). El RSEQ es una escala autoaplicada que evalúa el nivel de autoestima. Está formada por 10 items que son evaluados según una escala tipo Likert de 0 a 4 . Su puntuación máxima es 40 , reflejando las puntuaciones altas mayor nivel de autoestima. La consistencia del RSEQ en nuestro estudio fue $\alpha=0,86$.

\section{Instrumentos de evaluación de razonamiento causal}

Internal, Personal and Situational Attributions Questionnaire (IPSAQ; Kinderman y Bentall, 1996) El IPSAQ es un cuestionario que evalúa de forma explícita el estilo atribucional. Está basado en el Attributional Style Questionnaire (ASQ; Peterson et al., 1982); como éste, evalúa la dimensión internalidad (atribuir la causa de un suceso a uno mismo) y externalidad (atribuir la causa de un suceso a factores externos), pero además, el IPSAQ permite evaluar si la causa externa es atribuida a otras personas o a las circunstancias, solventando de esta forma los problemas de fiabilidad (Reivich, 1995) que presenta el ASQ en la subescala de externalidad. Está formado por 32 ítems que describen 16 situaciones hipotéticas positivas y 16 situaciones hipotéticas negativa (ver Apéndice I). La tarea del participante es indicar cuál podría ser la causa de cada una de las situaciones presentadas $\mathrm{y}$, además, valorar si dicha causa está relacionada consigo mismo (atribución interna), con otra /s persona/s (atribución externa personalizante) o con las circunstancias o el azar (atribución externa situacional). De esta forma, el IPSAQ informa del 
número total de atribuciones causales para sucesos positivos y negativos en tres subescalas. Los niveles de consistencia interna en nuestro estudio oscilaron entre $\alpha=0,57$ (atribuciones para sucesos positivos) y $\alpha=0,75$ (atribuciones para sucesos negativos). Las puntuaciones del IPSAQ permiten, además, el cálculo de dos sesgos cognitivos atribucionales: (1) Sesgo Externalizante (SE), obtenido mediante la sustracción del número total de atribuciones internas para sucesos negativos del número total de atribuciones internas para sucesos positivos - puntuaciones negativas indicarían la presencia de este sesgo; (2) Sesgo Personalizante negativo (SP) obtenido de la proporción del número de atribuciones externas personalizantes del total de atribuciones externas para sucesos negativos. Un valor superior a 0,5 reflejaría la presencia de sesgo personalizante. Estudios previos han mostrado que las propiedades psicométricas del IPSAQ son adecuadas (Day y Maltby, 2000).

Pragmatic Inferencial Test (PIT; Winters y Neale, 1985). El PIT es una tarea diseñada para evaluar de forma implícita (o encubierta) el estilo atribucional. Es presentada como una prueba de memoria con el fin de evitar la activación de sesgos cognitivos auto-protectores de forma consciente. Consta de 12 historias auto-referentes adaptadas de los ítems del ASQ (Peterson et al., 1982). Seis historias presentan sucesos hipotéticos positivos y 6 sucesos hipotéticos negativos. De cada una de ellas se derivan cuatro preguntas de elección múltiple. Sólo una de las respuestas es de tipo atribucional (evalúa locus de control interno vs. externo), mientras que las tres restantes hacen referencia a la historia presentada (ver Apéndice II). El PIT informa del número total de atribuciones causales en la escala de internalidad-externalidad para sucesos positivos y negativos. Además, permite obtener una puntuación compuesta denominada sesgo autosirviente (SA), definido como la tendencia a atribuir a causas internas sucesos positivos y a causas externas sucesos negativos. Esta puntuación es la resultante de la diferencia entre el número de atribuciones internas para suce- sos positivos y el número de atribuciones internas para sucesos negativos. Una puntuación negativa refleja menor sesgo autosirviente, es decir, una tendencia a atribuir a causas internas sucesos negativos y a causas externas sucesos positivos. El indice de consistencia, como ya ha sucedido en investigaciones anteriores (p.ej., Krstev et al., 1999) fue bajo: $\alpha=0,16$ para sucesos negativos y $\alpha=0,36$ para positivos.

\section{Procedimiento}

La participación de los pacientes en el estudio fue voluntaria, tras ser informados previamente de los objetivos del estudio y firmar un consentimiento informado. Cada uno de los participantes fue entrevistado en su hospital de referencia en un despacho privado por dos psicólogos entrenados previamente en la aplicación de las pruebas. El orden de presentación del protocolo de evaluación fue el mismo para todos los pacientes. En primer lugar se realizó la evaluación clínica y posteriormente se aplicaron las pruebas de evaluación de razonamiento causal. Puesto que el PIT es una medida implícita de razonamiento causal, esta tarea fue presentada antes de evaluar el estilo atribucional explícitamente con el IPSAQ.

\section{Análisis de datos}

La principal estrategia seguida fue el Análisis de Covarianza para determinar diferencias intergrupo en el caso de variables cuantitativas y pruebas Chi-Cuadrado en el caso de variables cualitativas. El nivel de significación asumido fue 0,05. El análisis estadístico de los datos se realizó empleando el paquete estadístico SPSS v. 12.0.

\section{RESULTADOS}

\section{Características clínicas}

Siguiendo la clasificación determinada por la entrevista estructurada del MADS, el gru- 
po de pacientes con delirios paranoides estuvo formado por 19 participantes, 11 hombres y 8 mujeres, y el grupo de pacientes con delirios no paranoides estuvo compuesto por 21 participantes, 16 hombres y 5 mujeres. En la Tabla 1 se muestran los estadísticos descriptivos de las variables socio-demográficas y clínicas de ambos grupos. No se encontraron diferencias significativas entre los grupos $\mathrm{ni}$ en género $\left[\chi^{2}(1,39)=1,484 ;\right.$ n.s. $]$, ni en nivel de educación $\left[\chi^{2}(1,39)=0,090 ;\right.$ n.s. $]$ ni en situación laboral $\left[\chi^{2}(1,39)=0,010 ; n . s.\right]$. Tampoco hubo diferencias entre los grupos en edad $[t(38)=0,792 ; n . s$.$] , ni en la edad de$ comienzo de la enfermedad $[\mathrm{t}(38)=-1,255$; n.s.]. En cuanto a duración de la enfermedad, los análisis estadísticos sí mostraron diferencias $[\mathrm{t}(38)=2,36 ; p<0,05]$, siendo la duración media del trastorno en el grupo no paranoide superior a la del grupo paranoide (ver Tabla 1). Por esta razón, la variable duración de la enfermedad fue introducida en todos los análisis estadísticos como covariable, a fin de controlar sus posibles efectos en los resultados obtenidos en la evaluación de la gravedad psicótica, el nivel de depresión, la autoestima y en el patrón de razonamiento causal (explícito e implícito).
Se realizaron ANCOVAs sucesivos de un factor (Grupo) para cada uno de los factores evaluados mediante el BPRS con el fin de determinar si había diferencias inter-grupo respecto a la gravedad psicopatológica. Los resultados de cada uno de los análisis indicaron la ausencia de diferencias significativas entre el grupo paranoide y el grupo no paranoide, siendo ambos grupos homogéneos en cuanto a la gravedad psicótica. En la Tabla 2 se muestra los resultados.

Análisis similares fueron efectuados para el nivel de depresión y autoestima. Los resultados indicaron que no había diferencias significativas entre los grupos en estas variables y que, por lo tanto, la gravedad clínica de los participantes era semejante (ver Tabla 2).

\section{Patrón de razonamiento causal explícito evaluado con el IPSAQ}

Se efectuaron tres ANCOVAs sucesivos de dos factores (Grupo: paranoide vs no paranoide $\times$ Tipo de Suceso: positivo vs negativo) con medidas repetidas en el segundo factor para cada una de las tres variables dependientes referentes al locus atribucional evaluado mediante

Tabla 1. Estadísticos descriptivos de las variables sociodemográficas y clinicas del grupo paranoide y del grupo no paranoide

\begin{tabular}{lrr}
\hline & & Grupo \\
& \multicolumn{1}{c}{$\begin{array}{c}\text { Naranoide } \\
\text { Variables sociodemográficas y clínicas }\end{array}$} & $\mathrm{N}=19$ \\
\hline Género & & 21 \\
$\quad$ Hombres & $11(57,9 \%)$ & $16(76,2 \%)$ \\
$\quad$ Mujeres & $8(42,1 \%)$ & $5(23,8 \%)$ \\
Edad media (DT) & $32,21(9,42)$ & $34,33(7,48)$ \\
Nivel de educación & & \\
$\quad$ Estudios primarios (EGB) & $1(5,3 \%)$ & $2(9,5 \%)$ \\
$\quad$ Estudios secundarios & $14(73,7 \%)$ & $15(71,4 \%)$ \\
$\quad$ Estudios superiores & $4(21,1 \%)$ & $4(19 \%)$ \\
Situación laboral & & $1(4,8 \%)$ \\
$\quad$ Nunca ha trabajado & $4(21,1 \%)$ & $12(57,1 \%)$ \\
$\quad$ Inactivo $>1$ año & $6(31,6 \%)$ & $6(28,6 \%)$ \\
$\quad$ Inactivo < l año & $7(36,8 \%)$ & $2(9,5 \%)$ \\
$\quad$ Estudiante & $2(10,5 \%)$ & $23,76(6,67)$ \\
Edad media de inicio de la enfermedad (DT) & $26,78(8,55)$ & $10,57(7,34)$ \\
Duración media de la enfermedad (DT) & $5,42(6,31)$ & \\
\hline
\end{tabular}


Tabla 2. Resultados evaluación clínica. Estadísticos descriptivos del grupo paranoide y no paranoide

\begin{tabular}{lrrr}
\hline & \multicolumn{3}{c}{ Grupos } \\
\cline { 2 - 4 } & $\begin{array}{c}\text { Paranoide } \\
(\mathrm{N}=19)\end{array}$ & $\begin{array}{c}\text { No Paranoide } \\
(\mathrm{N}=21)\end{array}$ & \\
Vedia (DT) & Media (DT) & \\
Variables clínicas & & & \\
\hline Gravedad psicopatológica (BPRS) & & & \\
$\quad$ Factor I: Ansiedad-Depresión & $7,63(2,69)$ & $8,09(3,89)$ & 0,$71 ;$ n.s. \\
Factor II: Anergia & $5,26(2,32)$ & $5,38(2,06)$ & 0,$05 ;$ n.s. \\
Factor III: Trastornos del pensamiento & $11,52(4,81)$ & $14,80(4,46)$ & 4,$00 ;$ n.s. \\
Factor IV: Activación & $3,73(1,75)$ & $3,71(1,30)$ & 0,$24 ;$ n.s. \\
Factor V: Hostilidad-Suspicacia & $7,52(3,43)$ & $6,28(3,01)$ & 1,$14 ;$ n.s. \\
Factor Síntomas Positivos & $22,78(7,05)$ & $24,80(5,97)$ & 0,$61 ;$ n.s. \\
Factor Síntomas Negativos & $5,62(2,32)$ & $5,38(2,06)$ & 0,$05 ;$ n.s. \\
Puntuación Total & $46,15(10,65)$ & $48,15(9,67)$ & 0,$46 ;$ n.s. \\
Nivel de Depresión (BDI) & $9,52(8,28)$ & $11,52(10,17)$ & 1,$15 ;$ n.s. \\
Autoestima (RSEQ) & $32,21(3,97)$ & $30,76(5,77)$ & 1,$10 ;$ n.s. \\
\hline
\end{tabular}

el IPSAQ. En primer lugar, respecto al Número total de atribuciones internas el ANCOVA $2 \times 2$ mostró que ni el efecto de la interacción Grupo $\times$ Tipo de suceso, ni el efecto del Grupo eran significativos. Sí fue significativo el Tipo de suceso $[\mathrm{F}(1,37)=16,39, p<0,05]$, siendo significativamente mayor la puntuación media del número total de atribuciones internas para sucesos positivos (media $=7,15 ; \mathrm{DT}=1,92$ ) que para sucesos negativos (media $=5,07 ; \mathrm{DT}=$ 3,48 ) realizadas por ambos grupos. En la Tabla 3 se muestran los estadísticos descriptivos y los resultados de estos análisis.

En segundo lugar, en el ANCOVA $2 \times 2$ (Grupo $\times$ Tipo de suceso) para el Número total de atribuciones externas-personalizantes ni el efecto de la interacción Grupo $\times$ Tipo de suceso ni el efecto del Grupo fueron significativos (ver Tabla 3). Sólo fue significativo el efecto del Tipo de suceso $[F(1,37)=7,26, p<0,05]$, siendo mayor el número de atribuciones externas-personalizantes de ambos grupos ante

Tabla 3. Resultados de la evaluación de razonamiento causal con tareas explícitas (IPSAQ) e implícitas (PIT)

\begin{tabular}{llcc}
\hline & & \multicolumn{2}{c}{ Grupos } \\
\cline { 3 - 4 } $\mathrm{N}^{\circ}$ total de atribuciones & & $\begin{array}{c}\text { Paranoide }(\mathrm{N}=19) \\
\text { Media (DT) }\end{array}$ & $\begin{array}{c}\text { No Paranoide (N =21) } \\
\text { Media (DT) }\end{array}$ \\
\hline Evaluación explícita (IPSAQ) & Sucesos positivos & & \\
& Internas & $7,42(2,06)$ & $6,90(1,81)$ \\
& Externas-personalizantes & $2,78(1,84)$ & $4,61(2,41)$ \\
& Externas-situacionales & $5,78(2,43)$ & $4,47(2,22)$ \\
& Sucesos negativos & & \\
& Internas & $5,84(3,94)$ & $4,38(2,94)$ \\
& Externas-personalizantes & $6,31(3,85)$ & $8,52(3,21)$ \\
& Externas-situacionales & $3,78(2,32)$ & $3,09(2,16)$ \\
& Sesgo externalizante & $1,57(3,54)$ & $2,52(3,24)$ \\
& Sesgo personalizante & $0,59(0,22)$ & $0,73(0,16)$ \\
& Internas & & \\
& Sucesos positivos & $2,52(1,02)$ & $3,04(1,35)$ \\
& Sucesos negativos & $2,94(1,26)$ & $2,19(1,07)$ \\
& Sesgo autosirviente & $-0,42(1,71)$ & $0,85(1,65)$ \\
\hline
\end{tabular}


sucesos negativos (media $=7,47 ; \mathrm{DT}=3,66$ ) que ante sucesos positivos (media $=3,75 ; \mathrm{DT}$ $=2,32$ ).

Finalmente, el ANCOVA $2 \times 2$ para Número total de atribuciones externas-situacionales reflejó que sólo el Tipo de suceso era significativo $[F(1,37)=13,40, p<0,05]$ siendo significativamente mayor el número de atribuciones externas situacionales ante sucesos positivos (media $=5,10 ; \mathrm{DT}=2,39$ ) que ante sucesos negativos (media $=3,42 ; \mathrm{DT}=2,24$ ) en ambos grupos (ver Tabla 3).

Respecto a los dos índices de sesgos cognitivos atribucionales, dos ANCOVAs de un factor (Grupo) para las variables sesgo externalizante (SE) y sesgo personalizante (SP) revelaron la ausencia de diferencias significativas entre el grupo paranoide y el grupo no paranoide - ver Tabla 3. La correlación entre estos dos índices no fue significativa ( $\mathrm{r}$ de Pearson $=-0,01, p=n . s$.).

\section{Patrón de razonamiento causal implícito evaluado con el PIT}

El PIT ofrece puntuaciones para la dimensión externalidad-internalidad tanto para sucesos positivos como para sucesos negativos (ver sección de Instrumentos). Puesto que estas puntuaciones son mutuamente excluyentes entre sí y no hubo datos perdidos, sería redundante informar de los resultados en ambas subescalas. Así, y con el fin de poder comparar posteriormente el patrón de razonamiento causal implícito con el obtenido con la evaluación explícita, se informa sólo de las puntuaciones obtenidas por los grupos en la subescala de internalidad (ver Tabla 3 ). Con el fin de estudiar el patrón de razonamiento causal implícito de cada grupo en función del tipo de suceso presentado, se realizó un ANCOVA de dos factores (Grupo $x$ Tipo de Suceso) con medidas repetidas en el segundo factor para el número total de atribuciones internas realizadas ante sucesos positivos y negativos. Los resultados mostraron que ni el efecto del Tipo de Suceso, ni el efecto de la variable Grupo fueron significativos. Por el contrario, el efecto de la interacción entre las variables Grupo y Tipo de Suceso fue significativo $[\mathrm{F}(1,37)$ $=4,79 ; p<0,05]$.

Análisis post-hoc indicaron que el número de atribuciones internas ante sucesos negativos era significativamente mayor en el grupo paranoide (media $=2,94$ ) que en el grupo no paranoide $($ media $=2,19)-$ DMS de Tukey $(38)=$ 0,$54 ; p<0,05$. Asimismo, el número de atribuciones internas del grupo no paranoide fue significativamente mayor ante sucesos positivos (media $=3,04)$ que ante sucesos negativos $($ media $=2,19)-$ DMS de Tukey $(38)=0,54 ; p$ $<0,05$. No se encontraron diferencias en el resto de los contrastes.

Respecto al índice de sesgo autosirviente (SA), un ANCOVA de un factor (Grupo) mostró la existencia de diferencias significativas entre los grupos $[F(1,37)=4,79 ; p<0,05]$, siendo mayor la puntuación media obtenida por el grupo no paranoide que por el grupo paranoide (ver Tabla 3). Así pues, el grupo no paranoide mostraba un sesgo atribucional implícito de naturaleza más positiva o autoprotectora que el grupo paranoide.

\section{Patrón de razonamiento causal explícito (IPSAQ) versus implícito (PIT)}

En la Figura 1 se muestran las puntuaciones obtenidas por el grupo paranoide y el grupo no paranoide en la dimensión de internalidad cuando ésta fue evaluada tanto con medidas explícitas (IPSAQ) como implícitas (PIT). Resulta de interés la comparación de ambas gráficas, pues muestra claras diferencias entre ambos grupos de participantes. El estilo de respuesta en el IPSAQ fue similar en ambos grupos, esto es, mostraron una mayor tendencia a atribuir a causas internas los sucesos positivos. Sin embargo, esta tendencia fue totalmente inversa sólo en el grupo de delirantes paranoides cuando se empleó una tarea implícita de razonamiento causal (i.e. PIT). Mientras que el comportamiento del grupo no paranoide permaneció estable, el grupo paranoide mostró una mayor tendencia a atribuir a causas internas los sucesos 
negativos, aún no siendo significativa la diferencia entre el número de atribuciones internas ante sucesos positivos y ante sucesos negativos como indicaron los contrastes post-hoc.

\section{DISCUSIÓN}

El objetivo general de nuestro trabajo consistió en comprobar si el modo de evaluación del estilo atribucional, mediante estrategias explícitas e implícitas, permitía detectar patrones atribucionales específicos en delirios paranoides. Nuestra hipótesis inicial de que la existencia de un sesgo externalizante y personalizante en estos pacientes se manifestaría sólo bajo una evaluación explícita y no implícita ha sido confirmada por nuestros resultados. En efecto, cuando se emplearon tareas explícitas, los pacientes paranoides mostraban un estilo de razonamiento causal externo semejante al encontrado en población normal (véanse las revisiones de Sweeney, Anderson y Bailey, 1986; Mezulis, Abramson, Hyde y Hankin, 2004).
El instrumento empleado (IPSAQ) permite un análisis más detallado y clínicamente relevante del locus atribucional puesto que permite diferenciar, dentro de las atribuciones externas, entre aquellas de índole situacional (p.ej. culpar a circunstancias) y aquellas otras que suponen una inculpación de otras personas (i.e., tendencia personalizante). Los resultados hallados muestran que en ambos tipos de delirio existe una tendencia significativa a acusar o culpabilizar a otras personas de los sucesos negativos hipotéticos descritos en las tareas empleadas aunque, en contra de los esperado, el sesgo personalizante no fue de mayor magnitud en el grupo paranoide. En cualquier caso, el hallazgo de la ausencia de diferencias entre los grupos es semejante al encontrado por Martin y Penn (2002). El hallazgo de un sesgo personalizante en pacientes delirantes confirma resultados previos en los que se ha analizado el patrón atribucional en el IPSAQ (p.ej. Kinderman y Bentall, 1997) y a la vez supone una contribución adicional pues detecta este mismo sesgo en pacientes con una temática delirante principal no per-

Total atribuciones internas

Evaluación explícita (IPSAQ)

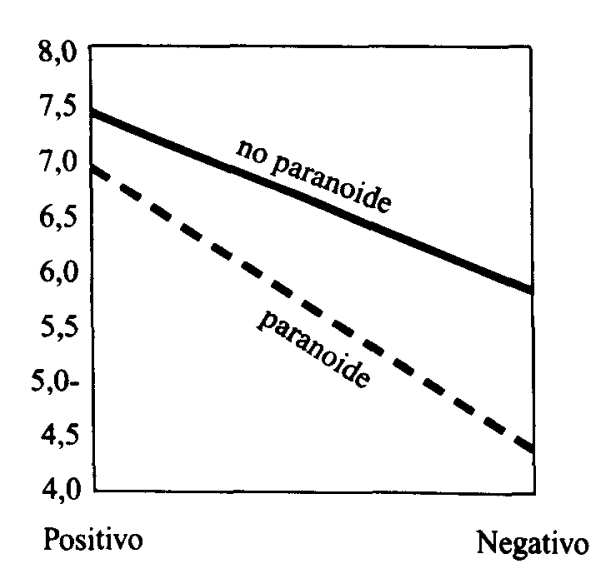

TIPO DE SUCESO
Evaluación implícita

(PIT)

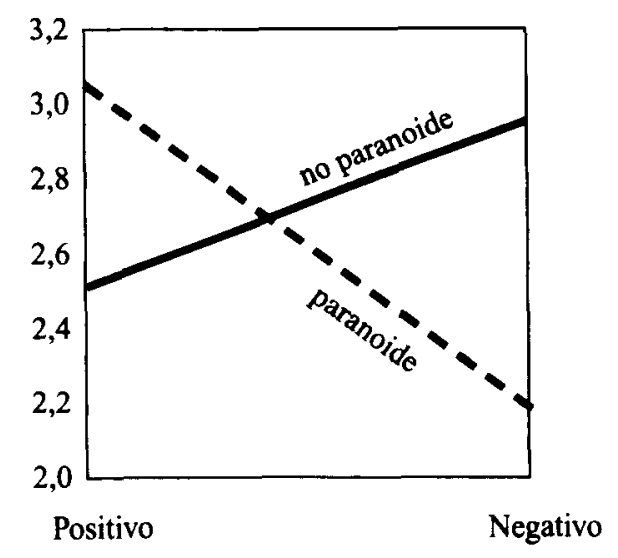

TIPO DE SUCESO

Figura 1. Comparación de las puntuaciones en la subescala de internalidad para sucesos positivos y negativos en el IPSAQ (atribuciones explícitas) y en el PIT (atribuciones implicitas) de los participantes con delirios paranoides y con delirios no paranoides. 
secutoria. Esta ausencia de diferencias en un producto cognitivo de tanta importancia potencial en la génesis y/o mantenimiento del delirio como lo son las atribuciones causales (Belloch, Sandin y Ramos, 1995; Bentall, Corcoran, Howard, Blackwood y Kinderman, 2001) emerge también en otros productos como las correlaciones ilusorias (Chadwick y Taylor, 2000), aunque cabe señalar que en otros productos cognitivos, como el recuerdo de material amenazante, sí se ha encontrado especificidad ligada al contenido persecutorio (p.ej. Kaney, Wolfenden, Dewey y Bentall, 1992).

En cualquier caso, puede parecer una obviedad indicar la gran importancia que puede tener un sesgo personalizante en el ámbito clínico. En efecto, la inculpación a otros puede tener muchas repercusiones clínicas en estos pacientes (p.ej. sentimientos de ira y de agravio, juicios erróneos sobre la intencionalidad, conductas de evitación pero también de agresión, o incapacidad para perdonar). La dificultad para encontrar $u$ ofrecer explicaciones situacionales o circunstanciales ante sucesos negativos puede ser de gran importancia en la configuración de un modelo de delirio paranoide (Bentall, 2003; Freeman et al., 2004). En cualquier caso, la investigación sobre estos sesgos atribucionales es aún muy escasa, a pesar de su gran relevancia, debido a la predominancia de un modelo conceptual atribucional que, siendo quizás un tanto simplista (v.g. locus externo vs. interno), ha dominado el panorama de la investigación en Psicología clínica.

El empleo de una tarea implícita para evaluar atribuciones causales hizo emerger un patrón muy interesante de resultados. Cuando se evaluó de modo encubierto el estilo atribucional, los pacientes no paranoides siguieron manteniendo un estilo de razonamiento causal que probablemente puede entenderse como un clásico mecanismo protector de la autoestima (Greenwald y Pratkanis, 1987) semejante al que se encuentra con una evaluación explícita (es decir, las atribuciones causales para sucesos negativos están caracterizadas por una menor internalidad que las explicaciones para sucesos positivos). Sin embargo, el patrón de razona- miento causal hallado en los paranoides varía en estas condiciones encubiertas. En comparación con los delirantes no paranoides, efectuaron más atribuciones internas para sucesos negativos. Para algunos autores (p.ej. Bentall, 2003) este tipo de hallazgos parecerian confirmar la presencia de autoesquemas negativos implícitos -de hecho, similares a los hallados en la depresión- y a la posible función autoprotectora del delirio paranoide. En situaciones en las que el individuo no percibe la necesidad de poner en marcha estos mecanismos defensivos, se facilitaría la posibilidad (como probablemente sucede en una tarea como el PIT) de la aparición de dichos autoesquemas cognitivos más negativos. No obstante, esta seductora idea necesita de más estudios en los que se utilicen diferentes medidas de cogniciones implícitas (ver revisión de tareas implícitas en Fazio y Olson, 2003; McKay et al., 2006) pero también de más estudios que repliquen los hallazgos dada la inconsistencia de los mismos en la literatura existente (p.ej. Lyon et al., 1994; Krstev, Jackson y Maude, 1999) que, en parte, pueden deberse a la escasa consistencia psicométrica de los instrumentos empleados, incluyendo el PIT (Díez-Alegría et al., en prensa). Una interesante alternativa puede ser, en nuestra opinión, el uso del Implicit Association Test (McKay, Langdon, y Colheart, 2006), una medida de una notable consistencia en la evaluación de actitudes implícitas.

El patrón de datos hallado no puede justificarse por diferencias sociodemográficas ni clínicas entre los grupos participantes. De hecho, los datos en medidas de gravedad psicopatológica, depresión, e incluso en autoestima confirman la comparabilidad psicopatológica de ambos grupos. No obstante, el patrón de razonamiento causal fue diferente. Investigaciones futuras habrán de determinar si este patrón se confirma en diferentes subtipos de ideas persecutorias. Por ejemplo, es posible que la distinción de Trower y Chadwick (1995) entre los paranoides «poor-me», (quienes no se creen merecedores de persecución), y los «bad-me» (quienes si se creen merecedores) puede ser relevante en este sentido pues los mecanismos 
mediadores de autoestima pueden ser muy diferentes en ambos casos (Freeman y Garety, 2000; Peters y Gartey, 2005). Melo et al. (2005) han sugerido que el «bad-me» y "poor me» representan dos fases de un ciclo. Seria de interés examinar qué procesos intervienen para pasar de una a otra fase y cómo está involucrada la emoción en estos cambios y su relación con el patrón de razonamiento causal.

En este trabajo hemos clasificado el tipo de delirio en función del tema predominante en su discurso psicopatológico atendiendo a diferentes dimensiones (grado de convicción, preocupación o resistencia al reto). Es posible que otros métodos puedan ofrecer diferentes agrupaciones de pacientes (e.g. basados en puntos de corte en gravedad psicótica mediante, por ejemplo, escalas como el BPRS) — ver estudio de Martin y Penn, 2002-, pero creemos que el empleo de esta estrategia dimensional responde a concepciones más actuales (aún habiendo sido planteadas hace décadas) -Strauss, 1969-, más adecuadas y menos categoriales del delirio que pueden arrojar luz sobre procesos subyacentes (Vázquez et al., 1999). No obstante reconocemos que los temas y contenidos delirantes, y en especial los de tipo persecutorio, son transversales. De hecho, con instrumentos como el SCAN-PSE se pueden detectar contenidos delirantes persecutorios en la mayoría de los participantes aunque la idea delirante principal del paciente no sea, medida con el MADS, de tipo persecutorio (v. Díez-Alegría et al., 2006).

Finalmente, nos gustaría destacar las implicaciones que estudios como el aquí planteado, desde el ámbito de la psicopatología clínica experimental, pueden tener en la práctica clínica. Los síntomas psicóticos positivos (i.e. delirios y alucinaciones) están mostrando ser susceptibles de intervención psicológica (p.ej. Cuevas-Yust y Perona-Garcelán, 1997; Valiente, 2002; Nieto y Sotoca, 2005), siendo las intervenciones cognitivo-conductuales eficaces en su tratamiento (p.ej. Haddock, Tarrier, Spaulding, Yusupoff, Kinney y McCarthy, 1998; Garety, Fowler y Kuipers, 2000) y en la mejoría del funcionamiento general de estos pacientes (Cormac, Jones, Campbell y Silveira da Mota Neto, 2004). Aunque aún no se conocen los mecanismos de cambio implicados, es posible que algunos factores ligados al procesamiento de la información (i.e. procesos atencionales, estilo atribucional, elaboración de conclusiones, etc.) - López-Luengo y Vázquez (2005)- puedan ser elementos mediadores importantes y de ahí la necesidad del apoyo de estudios básicos de dichos procesos que permitan avanzar en un campo, como el del delirio, relativamente ignorado en la investigación clínica experimental.

\section{REFERENCIAS}

American Psychiatric Association (1996). Manual diagnóstico y estadistico de los trastornos mentales, (4. ${ }^{\mathrm{a}}$ edición). Barcelona: Masson.

Beck, A. T., Rush, A. J., Shaw, B. F., y Emery, G. (1979). Cognitive therapy of Depression. New York: Guilford Press.

Belloch, A., Sandín, B. y Ramos, F. (Eds.) (1995). Manual de Psicopatología (Vol.1). Madrid: McGraw-Hill.

Bentall, R.P. (2003). Madness explained: Psychosis and human nature. London: Allen Lane.

Bentall, R.P., Corcoran, R., Howard, R., Blackwood, N. y Kinderman, P. (2001). Persecutory delusions: A review and theoretical integration. $\mathrm{Cli}$ nical Psychology Review, 21, (8), 1143-1 192.

Bentall, R.P. y Kaney, S. (1996). Abnormalities of self-representation and persecutory delusions: A test of a cognitive model of paranoia. Psychological Medicine, 26, 1231-1237.

Bentall, R.P., Kinderman, P. y Kaney, S. (1994). The self, attributional process and abnormal beliefs: towards a model of persectory delusionis. Behaviour Research and Therapy, 32, 331-341.

Brewin, C.R. (1985). Depression and causal attributions: What is their relation? Psychological Bulletin, 98, 297-309.

Buchanan, A., Reed, A., Wessely, S., Garety, P., Taylor, P., Grubin, D. y Dunn, G. (1993). Acting on delusions (2): the phenomenological correlates of acting on delusions. British Journal of Psychiatry, 163, 77-81.

Candido, C. y Romney, D. (1990). Attributional style in paranoid vs depressed patients. British Journal of Medical Psychology, 63, 355-363.

Chadwick, P. y Taylor, G. (2000). Are deluded people unusually prone to illusory correlation? Behavior Modification, 24, 130-141.

Cormac I, Jones C, Campbell C, Silveira da Mota Neto J. (2004). Cognitive behaviour therapy for 
schizophrenia (Cochrane Review). En: The Cochrane Library, Issue 3.Chichester: John Wiley \& Sons, Ltd.

Costello, C. (1992). Research on symptoms versus research on syndromes. Arguments in favour of allocating more research time to the study of symptoms. British Journal of Psychiatry, 160, 304-398.

Cuevas-Yust, C. y Perona-Garcelán, S. (1997). Tratamiento cognitivo-conductual de las ideas delirantes en un paciente esquizofrénico. Revista de Psicopatología y Psicología Clinica, 2, 275-291.

Day, L. y Maltby, J. (2000). Can Kinderman and Bentalls` suggestions for a personal and situational attributions questionnaire be used to examine all aspects of attributional style? Personality and Individual Differences, 29, 1047-1055.

Díez-Alegría, C., y Vázquez, C (en prensa). Teorías cognitivas del delirio. Apuntes de Psicologia.

Díez-Alegría, C., Vázquez, C., Nieto, M., Valiente, C. y Fuentenebro, F. (en prensa). Personalizing and externalizing biases in deluded and depressed patients: Are attributional biases a stable and specific characteristic of delusions? British Journal of Clinical Psychology.

Ey, H. (1950). Estudio sobre los delirios. Madrid: Paz Montalvo.

Fazio, R.H. y Olson, M.A. (2003). Implicit measures in social cognition research: their meaning and use. Annual Review of Psychology, 54, 297327.

Fear, C.F., Sharp, H. y Healy, D. (1996). Cognitive processes in delusional disorders. British Journal of Psychiatry, 168, 61-67.

Freeman, D. y Garety, P. (2000). Comments on the content of persecutory delusions: Does the definition need clarification? British Journal of Clinical Psychology, 39, 407-414.

Freeman, D., Garety, P.A., Fowler, D., Kuipers, E., Bebbington, P.E. y Dunn, G. (2004). Why do people with delusions fail to choose more realistic explanations for their experiences? An empirical investigation. Journal of Consulting and Clinical Psychology, 72, 4, 671-680.

Garety, P., Fowler, D. y Kuipers, E. (2000). Cognitive-behavioral therapy for medication-resistant symptoms. Schizophrenia Bulletin, 26, 73-86.

Greenwald, A. G., y Pratkanis, A. R. (1984). The self. En R. S. Wyer \& T. K. Srull (Eds.), Handbook of social cognition (pp. 129-178). Hillsdale, NJ: Erlbaum.

Haddock, G., Tarrier, N., Spaulding, W., Yusupoff, L., Kinney, C. y McCarthy, E. (1998). Individual cognitive-behaviour therapy in the treatment of hallucinations and delusions: A review. Clinical Psychology Review, 18, 821-838.
Kaney, S. y Bentall, R.P. (1989). Persecutory delusions and attributional style. British Journal of Medical Psychology, 62, 191-198.

Kaney, S., Wolfenden, M., Dewey, M. y Bentall, R. (1992). Persecutory delusions and the recall of threatening and non-threatening propositions. British Journal of Clinical Psychology, 31, 85-87.

Kelley, H.I. (1967). Attribution theory in social psychology. En d. Levine (Ed.), Nebraska Symposium on Motivation. Lincoln: University of Nebraska Press.

Kinderman, P. y Bentall, R.P. (1996a). A new measure of causal locus: The Internal, Personal and Situational Attributions Questionnaire. Personality and Individual Differences, 20, 261-264.

Kinderman, P. y Bentall, R.P. (1996b). Abnormalities of self-representation and persecutory delusions: a test of a cognitive model of paranoia. Psychological Medicine, 26, 1231-1237.

Kinderman, P. y Bentall, R.P. (1996c). Self-discrepancies and persecutory delusions: Evidence for a defensive model of paranoid ideation. Journal of Abnormal Psychology, 105, 106-114.

Kinderman, P. y Bentall, R.P. (1997). Causal attributions in paranoia and depression: internal, personal, and situational attributions for negative events. Journal of Abnormal Psychology, 106, 341-345.

Krstev, H., Jackson, H. y Maude, D. (1999). An investigation of attributional style in first-episode psychosis. British Journal of Clinical Psycho$\log y, 38,181-194$.

Levenson, H. (1974). Activism and powerful others: Distinctions within the concept of internal-external control. Journal of Personality Assesment, 38, 377-383.

López-Luengo, B. y Vázquez, C. (2005). Effects of a neuropsychological rehabilitation program on schizophrenic patients' subjective perception of improvement. Neuropsychological Rehabilitation, 15, 609-618.

Lyon, H., Kaney, S. y Bentall, R.P. (1994). The defensive function of persecutory delusions. Evidence from attributions tasks. British Journal of Psychiatry, 164, 637-646.

Lukoff, D., Nuechterlein, K.H., \& Ventura, J. (1986). Appendix A: Manual for the Expanded Brief Psychiatric Rating Scale (BPRS). Schizophrenia Bulletin, 12, 594-602.

Martin, J.A. y Penn, L. D. (2002). Attributional style in schizophrenia: An investigation in outpatients with and without persecutory delusions. Schizophrenia Bulletin, 28, 131-141.

McKay, R., Langdon, R., \& Coltheart, M. (2006, en prensa) The defensive function of persecutory delusions: An investigation using the Implicit Association Test. Cognitive Neuropsychiatry. 
McKay, R., Langdon, R. \& Coltheart, M. (2005). «Sleights of mind»: Delusions, defences and selfdeception. Cognitive Neuropsychiatry, 10, 305326.

Melo, S.S., Taylor, J.L. and Bentall, R.P. (2005). Poor me' versus 'bad me' paranoia and the instability of persecutory ideation. Psychology and Psychotherapy: Theory, Research and Practice. Sometido a revisión.

Mezulis, A.H., Abramson, L.Y., Hyde, J.S. y Hankin, B.J. (2004). Is there a universal positivity bias in attributions? A meta-analytic review of individual, developmental, and cultural differences in the self-serving attributional bias. Psychological Bulletin, 130, 711-747.

Nieto, M. y Sotoca, A. (2005). Trastorno delirante (tipo persecutorio). En J.P. Espada, J. Olivares y F.X. Méndez (Ed.), Terapia psicológica. Casos prácticos (pp. 299-327). Madrid: Pirámide.

Persons, J. (1986). The advantages of studying psychological phenomena rather than psychiatric diagnosis. American Psychologist, 41, 1252-1260.

Peters, E. y Garety, P. (2005, en prensa). Cognitive functioning in delusions: a longitudinal analysis. Behaviour Research and Therapy.

Peterson, C., Semmel, A., von Baeyer, C., Abramson, L.Y., Metalsky, G.I. y Seligman, M.E.P. (1982). The Attributional Style Questionnaire. Cognitive Therapy and Research, 6, 287-300.

Reivich, K. (1995). The measurement of explanatory style. En G. M. Buchanan y M.E.P. Seligman (Eds.), Explanatory style (pp. 71-98). Hillsdale, NJ: Erlbaum.

Rosenberg, M. (1965). Society and the adolescent self image. Princenton: Princenton University Press.

Sanz, J. y Vázquez, C. (1998). El inventario para la Depresión de Beck (BDI) como instrumento para identificar sujetos deprimidos y no deprimidos en la investigación psicopatológica: Fiabilidad, validez y datos normativos en muestras universitarias. Psicothema, 10, 303-318.

Sartorius, N., Jablensky, A., Korten, A., Ernberg, G., Andker, M., Cooper, J.E. y Day, R. (1986). Early manifestations and first-contact incidence of schizophrenia in different cultures. Psychological Medicine, 16, 909-928.

Smith, N., Freeman, D., \& Kuipers, E., (2005). Grandiose delusions: An experimental investigation of the delusion-as-defence hypothesis. Journal of Nervous and Mental Disease, 193, 480-487.

Strauss, J.S. (1969). Hallucinations and delusions as points on continua function. Archives of General Psychiatry, 20, 581-586.

Sweeney, P.D., Anderson, K. y Bailey, S. (1986). Attributional Style in Depression: A Meta-Analytic Review. Journal of Personality and Social Psychology, 50, 974-991.

Taylor, P., Garety, P., Buchanan, A., Reed, A., Wesseley, s., Ray, K., Dunn, G. y Grubin, D. (1994). Delusions and violence. En J. Monahan y H. Steadman (Eds.), Violence and mental disorder: developments in risk assesment. Chicago: Chicago University Press.

Trower, P. y Chadwick, P. (1995). Pathways to defence of the self: A theory of two types of paranoia. Clinical Psychology: Science and Practice, 2, 263-278.

Valiente, C. (2002). Alucinaciones y delirios. Madrid: Síntesis.

Vázquez, C. y Sanz, J. (1999). Fiabilidad y validez de la versión española del Inventario para la Depresión de Beck de 1978 en pacientes con trastornos psicológicos. Clínica y Salud, 10, 59-81.

Vázquez, C., Valiente, C. y Díez-Alegría, C. (1999). La evaluación del delirio: Desde los sistemas categoriales a la evaluación multidimensional. En F. Silva (Ed.), Avances en la Evaluación Psicológica (pp. 313-360). Valencia: Promolibro.

Winters, K.C. y Neale, J.M. (1985). Mania and low self-esteem. Journal of Abnormal Psychology, 94. 282-290.

White, P.A. (1991). Ambiguity in the internal / external distinction in causal attribution. Journal of Experimental Social Psychology, 27, 259-270.

Zigler, E. \& Glick, M. (1988). Is paranoid schizophrenia really camouflaged depression? American Psychologist, 43, 284-290. 


\section{APÉNDICE I. Ejemplos de sucesos hipotéticos positivos y negativos presentados en el IPSA}

1. Un amigo piensa que eres de confianza.

¿Cuál es la causa de que tu amigo piense que eres de confianza?

(Por favor, indique la principal causa)

\section{Esto es:}

a. ¿algo sobre ti?

b. ¿algo sobre la otra persona o la gente?

c. ¿algo sobre la situación (circunstancias o azar)?

2. Un amigo habla sobre ti a tus espaldas.

¿Cuál es la causa de que tu amigo hable sobre ti a tus espaldas?

(Por favor, indique la principal causa)

\section{Esto es:}
a. ¿algo sobre ti?
b. ¿algo sobre la otra persona o la gente?
c. ¿algo sobre la situación (circunstancias o azar)? 
APÉNDICE II. Ejemplos de sucesos hipotéticos positivos y negativos presentados en el PIT ( ${ }^{*}$ Indica la respuesta en la que se solicita una inferencia causal)

A. Una persona mayor y solitaria se sienta a tu lado en un banco del parque mientras estás leyendo un libro y empieza a hablar contigo. No te sorprende ya que los extraños suelen ser muy amables contigo. Después de una corta conversación, te das cuenta de que esta persona está pasando momentos dificiles y necesita ayuda. Habláis durante un buen rato, y te parece que esta persona sigue disfrutando de tu compañia.

A 1. ¿Quién empieza una conversación contigo?
a) un turista
b) un extraño

*A 2. ¿Por qué esta persona habla contigo tanto tiempo?
a) eres amable
b) quiere tu ayuda

A 3. ¿Qué estabas haciendo cuando llega esta persona?
a) leyendo el periódico
b) leyendo un libro

A 4. ¿Por qué está pasando esta persona momentos difíciles?
a) enfermedad
b) abandonado por su familia

B. Recientemente no has hecho todo el trabajo que tu jefe esperaba de ti. Tu jefe empieza a quejarse sobre tu actuación. El trabajo a veces es dificil para ti porque es bastante técnico y las horas son una carga. Además, recientemente descubres a través de un rumor en la oficina que el sobrino de tu jefe está muy interesado en conseguir tu puesto de trabajo.

B 1. ¿Con quién has hablado sobre tus problemas en el trabajo?
a) con nadie
b) con tu esposa

B 2. ¿Qué clase de habilidad requiere este trabajo?
a) manual
b) técnica

*B 3.¿Por qué se queja tu jefe sobre tu actuación en el trabajo?
a) tienes pocas habilidades técnicas
b) quiere que renuncies para hacer sitio a un pariente

B 4. ¿En qué turno trabajas?
a) de día
b) de noche 